\title{
Blonanserin Induced Hypokinesia and Tardive Dyskinesia
}

\section{Saraswathy $\mathbf{G R}^{1 *}$, Virupaksha $\mathbf{H S}^{2}$, Thejaswi $\mathbf{G}^{1}$ and Lakshmi Narayan $\mathbf{A}^{1}$}

${ }^{1}$ Department of Pharmacy Practice, FPH, MSRUAS, Bengaluru, Karnataka, India

${ }^{2}$ Department of Psychiatry, M.S. Ramaiah Medical College, Bengaluru, Karnataka, India

\begin{abstract}
A 27 year old male diagnosed with psychosis, who was treated with Risperidone followed by aripiprazole was reported to present with adverse effects. Hence the therapy was changed to Blonanserin. It is a dopamine serotonin antagonist which has got fewer propensities to cause tardive dyskinesia (TD) than conventional antipsychotics and other atypical antipsychotics. This is a report on blonanserin induced hypokinesia followed by TD in the form of perioral tremors in a mid-aged male patient diagnosed with psychosis. There have been no reports on TD induced by blonanserin. Clinicians must be aware of the possibility of the patients developing hypokinesia and TD when they are treated with the supposedly safe atypical antipsychotics like blonanserin.
\end{abstract}

Keywords: Blonanserin, Atypical antipsychotics; Tardive dyskinesia; Hypokinesia

\section{Introduction}

Tardive dyskinesia (TD) is one of the common adverse effects associated with the long-term use of neuroleptics, characterized by involuntary, choreiform, athetoid or rhythmic movements of the tongue jaw and / or extremities [1]. TD was reported in $20-30 \%$ of the general population under antipsychotic therapy [2]. It was hoped that the atypical antipsychotics such as, Risperidone, Olanzapine, Quetiapine, Ziprasidone and Blonanserin will have a reduced liability to produce TD. Herein, we report a case of TD induced by Blonanserin.

\section{Case Report}

A 27 year old male diagnosed with psychosis developed Extrapyramidal Symptoms (EPS) like tremors and rigidity within one month of Risperidone therapy. Trihexyphenidyl (THP) $4 \mathrm{mg}$ was employed to manage the EPS and the dose of THP was reduced to $2 \mathrm{mg}$ with the resolution of EPS. Though the psychotic symptoms and EPS resolved the patient developed erectile dysfunction due to which Risperidone therapy was tapered and discontinued, however, the patient was started on Aripiprazole. The patient developed severe akathisia after two weeks of initiation of treatment with Aripiprazole. Propranolol $20 \mathrm{mg}$ was prescribed for the management of akathisia but it did not resolve which resulted in discontinuation of Aripiprazole. Propranolol was still continued till the complete resolution of akathisia. The psychiatrist planned to start the antipsychotic therapy with olanzapine while the patient's family history of metabolic disorders made the option to be Blonanserin. The initial dose of Blonanserin was $4 \mathrm{mg} /$ day and gradually increased to $12 \mathrm{mg}$ /day. The patient was responding with Blonanserin with well controlled symptoms. The patient developed symptoms of hypokinesia like decreased arm swing, facial expressions and mild rigidity. THP dose was increased to $6 \mathrm{mg}$ per day in the view to manage hypokinesia. Two months later the patient presented with perioral tremors which was considered to be one of the symptoms of TD. Blonanserine induced hypokinesia and TD was suspected. Therefore, Blonanserine was tapered and discontinued. The patient was put on $10 \mathrm{mg}$ Olanzepine therapy in spite of his family history of diabetes mellitus. Olanzepine's dose was increased to $20 \mathrm{mg}$ which resulted in sedation, bilateral pedal edema. Olanzepine's dose was tapered to $5 \mathrm{mg}$ to resolve the edema while the patient developed psychotic symptoms, hence Olanzepine was increased to $10 \mathrm{mg}$ per day. The treatment was continued further and the patient responded to the therapy with well controlled symptoms.

\section{Discussion}

Blonanserin is a novel atypical antipsychotic drug with specific receptor binding characteristics on Dopamine-Serotonin receptors as antagonist. It is as effective as Haloperidol and Risperidone in the treatment of schizophrenia. It causes greater improvement of negative symptoms as compared to Haloperidol, while, EPS and sedation is lower than Haloperidol. Incidence of hyperprolactinemia, weight gain, increased appetite, and orthostatic hypotension is lower than Risperidone. Oral blonanserin is generally well tolerated in patients of schizophrenia with most adverse reactions being of mild to moderate severity $[3,4]$.

In this case we have observed Blonanserin induced hypokinesia and perioral tremors (TD) which is not reported elsewhere. The hypokinesia and perioral tremors were observed after two months of initiation of Blonanserin in this case. Based on Naranjo's algorithm for causality assessment, the reaction was found to be probable with the score of 6 . However, since the patient developed adverse drug reactions with other antipsychotics prior to Blonanserin, further evaluation is required to completely rule out the other causes of TD and to confirm that Blonanserin is responsible for inducing TD in a mid-aged male patient with psychosis.

\section{References}

1. Daniel DG, Egan MF, Wolf SS (2000) Neuropsychiatric aspects of movement disorders. In: Comprehensive Textbook of Psychiatry. (7thedn), Lippincott Williams \& Wilkins, Philadelphia, USA.

2. American Psychiatric Association (1994) Diagnostic and Statistical Manual for mental disorders. (4thedn), American Psychiatric Association, Washington DC, USA.

3. Murasaki M (2007) Clinical evaluation of blonanserin for schizophrenia: a double-blind trial comparing blonanserin with haloperidol. Jpn J Clin Psychopharmacol 10: 2059-2079.

4. Miura S (2008) Clinical evaluation of blonanserin for schizophrenia: a randomized study comparing blonanseria with risperidone. Jpn J Clin Psychopharmacol 11: 297-314.

*Corresponding authors: Saraswathy GR, Department of Pharmacy Practice, Faculty of Pharmacy, M.S. Ramaiah University of Applied Sciences Bangalore, Karnataka, India. Tel: 08123230400; Fax: 080-23607488; E-mail: saraswathypradish@gmail.com

Received October 06, 2015; Accepted October 27, 2015; Published October 30, 2015

Citation: Saraswathy GR, Virupaksha HS, Thejaswi G, Lakshmi Narayan A (2015) Blonanserin Induced Hypokinesia and Tardive Dyskinesia. J Mol Biomark Diagn 6 : 254. doi:10.4172/2155-9929.1000254

Copyright: (C) 2015 Saraswathy GR, et al. This is an open-access article distributed under the terms of the Creative Commons Attribution License, which permits unrestricted use, distribution, and reproduction in any medium, provided the original author and source are credited. 